\title{
Interventions to Improve Antiretroviral Therapy Adherence Among Adolescents and Youth in Low- and Middle-Income Countries: A Systematic Review 2015-2019
}

\author{
Lindsey K. Reif ${ }^{1,6}$. Elaine J. Abrams s,4,6 . Stephen Arpadi ${ }^{2,3,4}$ - Batya Elul ${ }^{6}$ - Margaret L. McNairy ${ }^{1,5}$. \\ Daniel W. Fitzgerald ${ }^{1} \cdot$ Louise Kuhn ${ }^{2,6}$
}

Published online: 9 March 2020

(c) The Author(s) 2020

\begin{abstract}
Adolescents and youth living with HIV have poorer antiretroviral treatment (ART) adherence and viral suppression outcomes than all other age groups. Effective interventions promoting adherence are urgently needed. We reviewed and synthesized recent literature on interventions to improve ART adherence among this vulnerable population. We focus on studies conducted in low- and middle-income countries (LMIC) where the adolescent and youth HIV burden is greatest. Articles published between September 2015 and January 2019 were identified through PubMed. Inclusion criteria were: [1] included participants ages 10-24 years; [2] assessed the efficacy of an intervention to improve ART adherence; [3] reported an ART adherence measurement or viral load; [4] conducted in a LMIC. Articles were reviewed for study population characteristics, intervention type, study design, outcomes measured, and intervention effect. Strength of each study's evidence was evaluated according to an adapted World Health Organization GRADE system. Articles meeting all inclusion criteria except being conducted in an LMIC were reviewed for results and potential transportability to a LMIC setting. Of 108 articles identified, 7 met criteria for inclusion. Three evaluated patient-level interventions and four evaluated health services interventions. Of the patient-level interventions, two were experimental designs and one was a retrospective cohort study. None of these interventions improved ART adherence or viral suppression. Of the four health services interventions, two targeted stable patients and reduced the amount of time spent in the clinic or grouped patients together for bi-monthly meetings, and two targeted patients newly diagnosed with HIV or not yet deemed clinically stable and augmented clinical care with home-based case-management. The two studies targeting stable patients used retrospective cohort designs and found that adolescents and youth were less likely to maintain viral suppression than children or adults. The two studies targeting patients not yet deemed clinically stable included one experimental and one retrospective cohort design and showed improved ART adherence and viral suppression outcomes. ART adherence and viral suppression outcomes remain a major challenge among adolescents and youth. Intensive home-based case management models of care hold promise for improving outcomes in this population and warrant further research.
\end{abstract}

Keywords Adolescent $\cdot$ Youth $\cdot$ ART adherence $\cdot$ Review $\cdot$ Intervention

\section{Introduction}

Adolescents and youth, 10 to 24 years of age, represent a growing proportion of people living with HIV around the world and have worse outcomes than all other age groups [1-6]. In recent years, AIDS-related deaths among

Lindsey K. Reif

lir2020@med.cornell.edu

Extended author information available on the last page of the article adolescents and youth increased by $50 \%$ while they have decreased among all other age groups [7]. In 2018, 510,000 young people between the ages of 10 to 24 years were newly-infected with HIV, $40 \%$ of whom were between 10 and 19 years of age [8]. In addition to heterosexual transmission, a generation of children infected with HIV perinatally are now aging into adolescence, adding to the burden of disease in this age group.

Adequate adherence to an antiretroviral therapy (ART) regimen leading to viral suppression is essential for an adolescents' own health and well-being, and to reduce further 
HIV transmission. Yet, adolescents and youth have poor adherence to drug regimens for many chronic illnesses [9-12]. Adherence to ART is further complicated by HIVrelated stigma [13-16]. The period of adolescence and youth is characterized as a time of great physiological and psychological growth and development [1, 17], increased desire for independence from parents [18], and increased risk-taking [19], adding another layer of complexity. During this developmental stage, initiation of sexual activity is common and may include early pregnancy [20]. Adolescents and youth also lack financial autonomy, are prone to peer pressure, and lack problem-solving skills [21-25]. Further, in resource-limited settings, external factors including poverty, food scarcity, and HIV-related stigma acutely influence ART adherence and HIV outcomes [26-33].

Major barriers to ART adherence for adolescents and youth can be divided into 3 categories: patient-level factors (e.g. socioeconomic status, stigma) [34, 35], health services factors (e.g. clinic waiting times, drug availability, quality of care) [36, 37], and medication factors (e.g. dosing, high pill burden, side effects) $[3,38,39]$. Much of the research on ART adherence among young people has focused on identifying and estimating the prevalence of these barriers [29, $33,38,40,41]$. A comprehensive review of the literature between 2003 and 2015 identified 10 studies which evaluated interventions to improve adherence in adolescents in developed countries [42]. Effective interventions included daily interactive text reminders for dosing [43, 44], and computer-driven support programs [45]. However, none of the studies included were conducted in a low- or middle-income country (LMIC) [46], areas where the global HIV epidemic is centered. Further, most of these studies were descriptive reports or pilot studies with small sample sizes and thus had insufficient power to detect meaningful effects.

Given the critical need to identify effective approaches to improve outcomes among adolescents and youth living with HIV, we evaluated and synthesized the recent published literature on research conducted in a LMIC aimed at improving ART adherence in this population.

\section{Methods}

\section{Article Search and Selection}

We searched the PubMed database for English language articles which evaluated interventions to improve ART adherence among adolescents and youth living with HIV, conducted in a LMIC, and published between September 2015 and January 2019 using the search terms indicated in Fig. 1 [47]. We then manually reviewed the references sections of relevant articles. Records were managed using EndNote and duplicates were removed manually. One reviewer (LR) conducted the primary search and articles selected for inclusion were approved by all authors. Articles selected met the following criteria: (1) included adolescents and youth ages 10-24 years; (2) evaluated an intervention to improve ART adherence; (3) included an ART adherence measurement
Fig. 1 Flowchart for articles published in PubMed between September 2015 and January 2019 which were reviewed under search term [(()((() structural) or ((behavioral) $))$ and intervention $))$ and $((((($ ART $)$ or HIV medication) or HAART)) and ((adherence) or persistence) $)$ and HIV)) ) and (((young adult) or adolescent) or adolescence) or youth))]

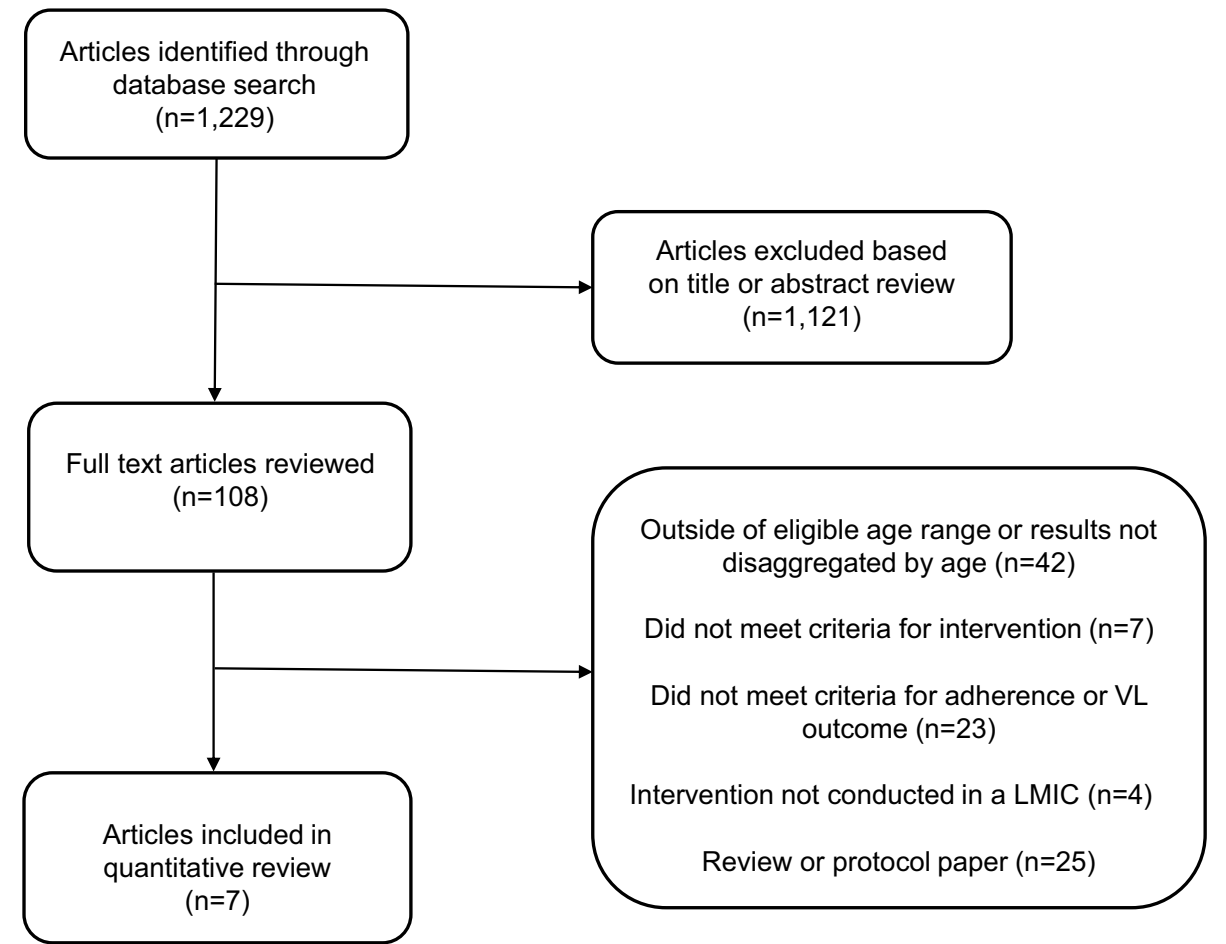


outcome (self-report, pill count, or medication event monitoring systems (MEMS)) or viral load (VL) as a proxy for an adherence measurement; (4) conducted in a LMIC. Eligible manuscripts were not restricted by study design and included studies which evaluated structural, behavioral, or health services-related interventions aimed at improving ART adherence.

\section{Data Extraction and Synthesis}

Each of the articles meeting our inclusion criteria was reviewed for characteristics of the study population (e.g. age, time on ART, health status at baseline), type of intervention evaluated, study design, outcome measured (ART adherence or VL), and intervention efficacy. The strength of each study's evidence was evaluated according to an adapted GRADE system utilized by the World Health Organization [48]. This system classifies studies into four levels based on study design, analysis plan, and existence of comparison groups. High quality evidence (level 4) are randomized control trials (RCT) with statistical testing comparing groups. Moderate quality evidence (level 3) are prospective cohort studies with statistical testing comparing groups. Low quality evidence (level 2) are retrospective or descriptive studies with statistical testing of between or within group comparisons; and very low quality evidence (level 1) are studies without statistical comparisons.

Due to the small selection of studies and diversity of outcome measures, a meta-analysis was not conducted to synthesize the effect size across studies. We reviewed the seven included studies to determine interventions with the most potential for impact and assessed areas in need of further research and evidence.

\section{Results}

\section{Included Studies and Populations}

The PubMed search identified 1229 articles, of which 1121 were excluded based on review of the abstract. A full text review was completed on 108 articles. Seven studies met the inclusion criteria. Reasons for exclusion are listed in Fig. 1; if an article was excluded for failing to meet more than one criteria, it is listed under the first exclusion category as prioritized in the criteria above. The most common reason for exclusion was only including participants outside the age range of interest (10-24 years) or reporting outcomes which were not disaggregated so as to discern results among adolescents and youth (i.e. results reported for ages 18-29 years). The seven studies meeting our inclusion criteria are described in Table 1. All were conducted in sub-Saharan Africa and included adolescents living with
HIV who were aware of their HIV status and were receiving HIV care at a health facility. The median sample size was 702 (range 94-96,706).

We also reviewed and report the results of 4 articles that were excluded because the studies were not conducted in a LMIC. We briefly summarize the results of these studies here and their potential transportability to an LMIC setting, but did not include them in the main review since our primary goal was to identify interventions which would be directly applicable in resource-limited settings where the global HIV burden among adolescents and youth is greatest.

\section{Intervention Types}

Studies either focused on: (1) patient-level interventions, i.e. interventions implemented at the individual level in addition to standard HIV care $(\mathrm{n}=3)$; or (2) health services interventions which re-structured the way HIV care was provided, also called 'models of HIV care' [49, 50] $(n=4)$.

In the first patient-level intervention, a once-weekly SMS text message was designed to check-in with participants about their general well-being [51]. One study arm received this weekly check-in message but could not respond to the sender. The second arm received the same message and could respond to the sender. The third study arm received standard care, i.e. no messages. The second study evaluated an economic intervention in which a savings account was established which could be used for small business development or education, i.e. school fees or lunches to address financial-related barriers to ART adherence [52]. In the third study, three consecutive monthly individual intensive adherence counseling sessions were provided to participants. The goal of the sessions was to identify adherence barriers and develop individualized plans to address them [53].

Four studies evaluated health services interventions which are categorized as either less intensive $(n=2)$ or more intensive $(n=2)$ models of care (Fig. 2). The two less intensive models of care reduced the amount of time stable participants spent in the clinic. In the first, multi-month ART prescriptions allowed participants to come to the clinic less frequently ranging from every other month and some only twice per year for medication refills and clinical check-ups [54]. In the second, a group-based model of care, patients formed groups of 25-30 participants and met every other month for group counseling, brief check-ups, and distribution of ART refills [55]. Groups were facilitated by a lay health worker and nurse and met at a health facility or community venue.

Two studies of more intensive models of care targeted newly diagnosed adolescents initiating ART or those who had not yet been defined as adherent or stable. These two models of care augmented regular care with additional support through home-based case management by a community 


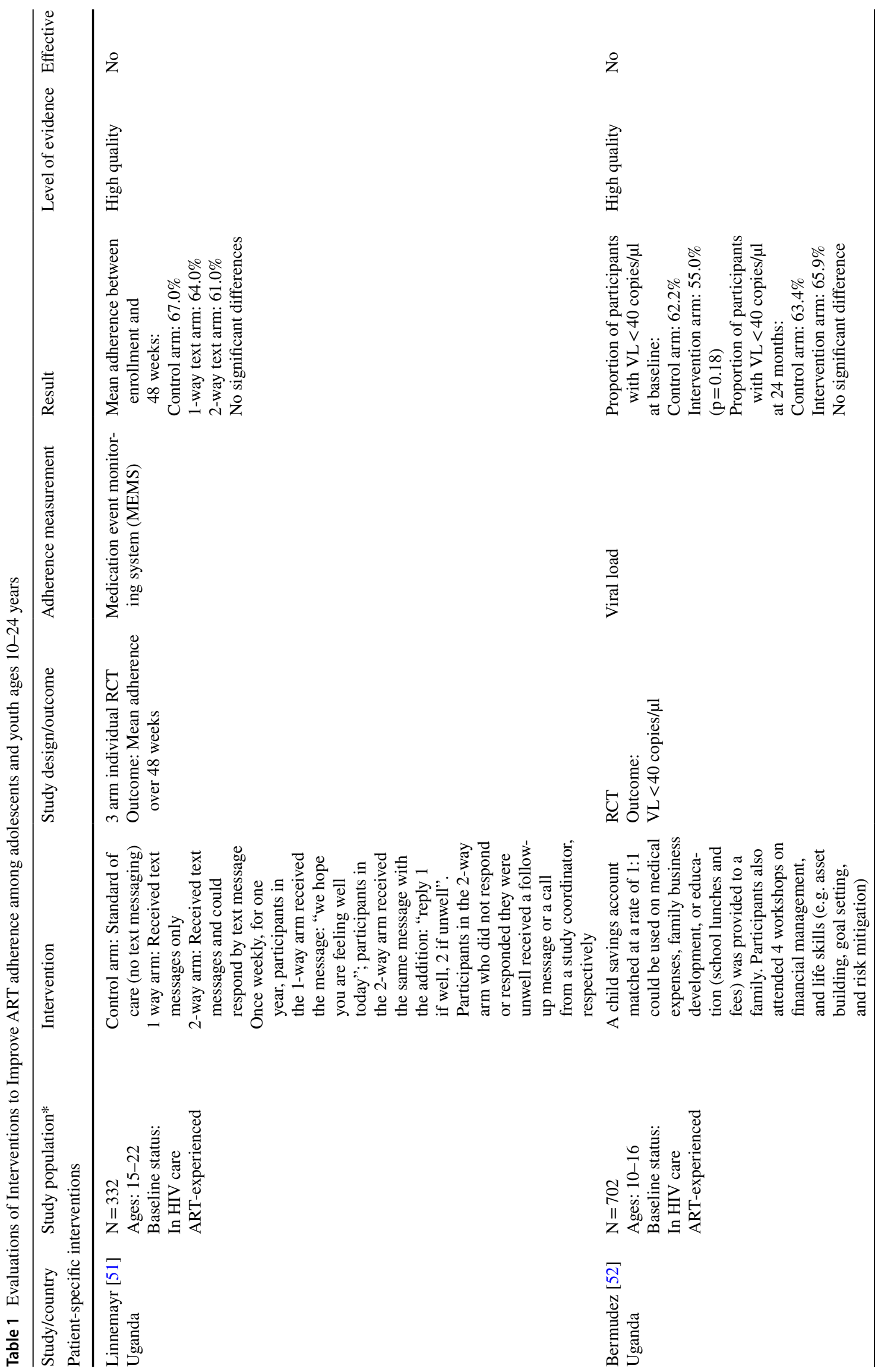




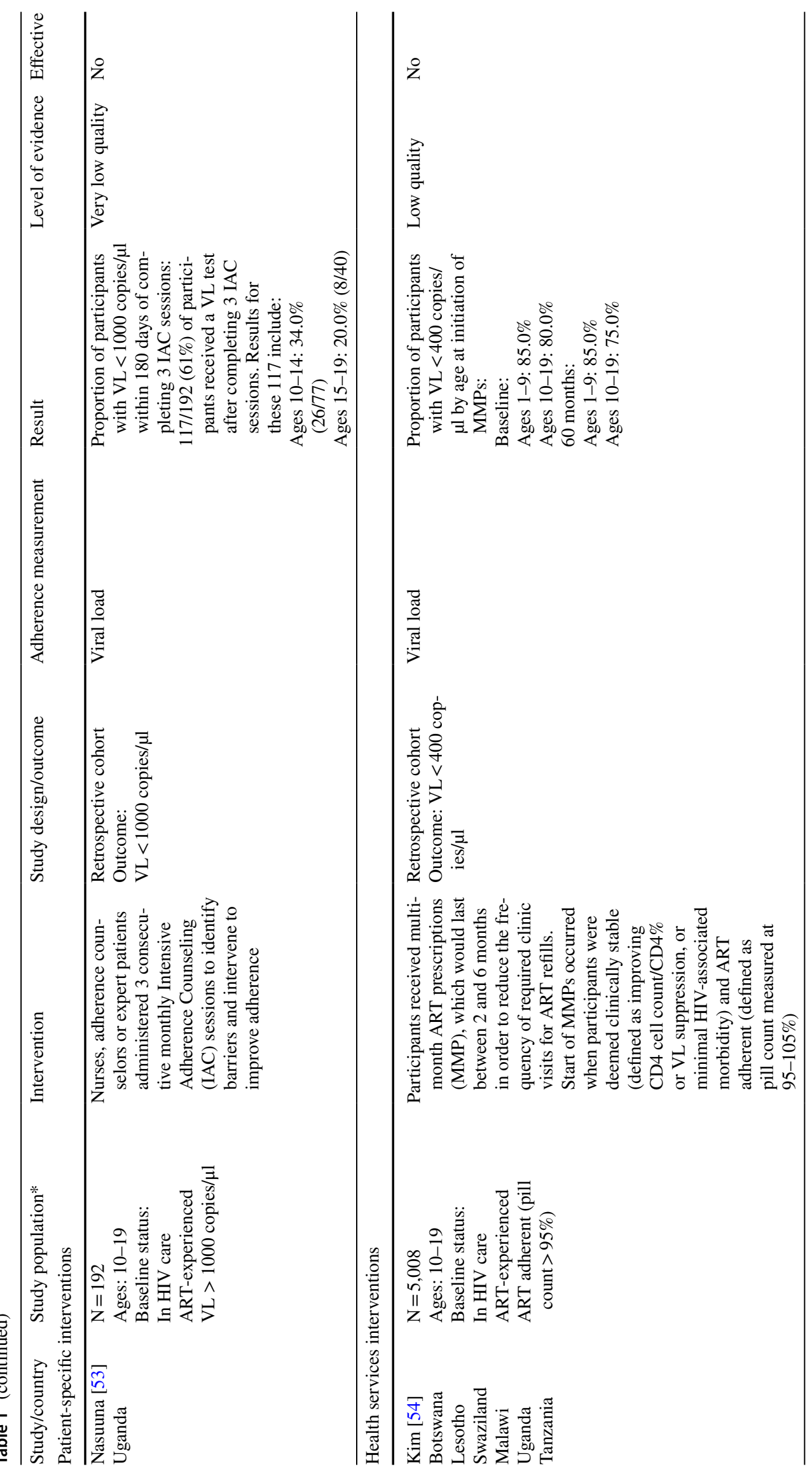




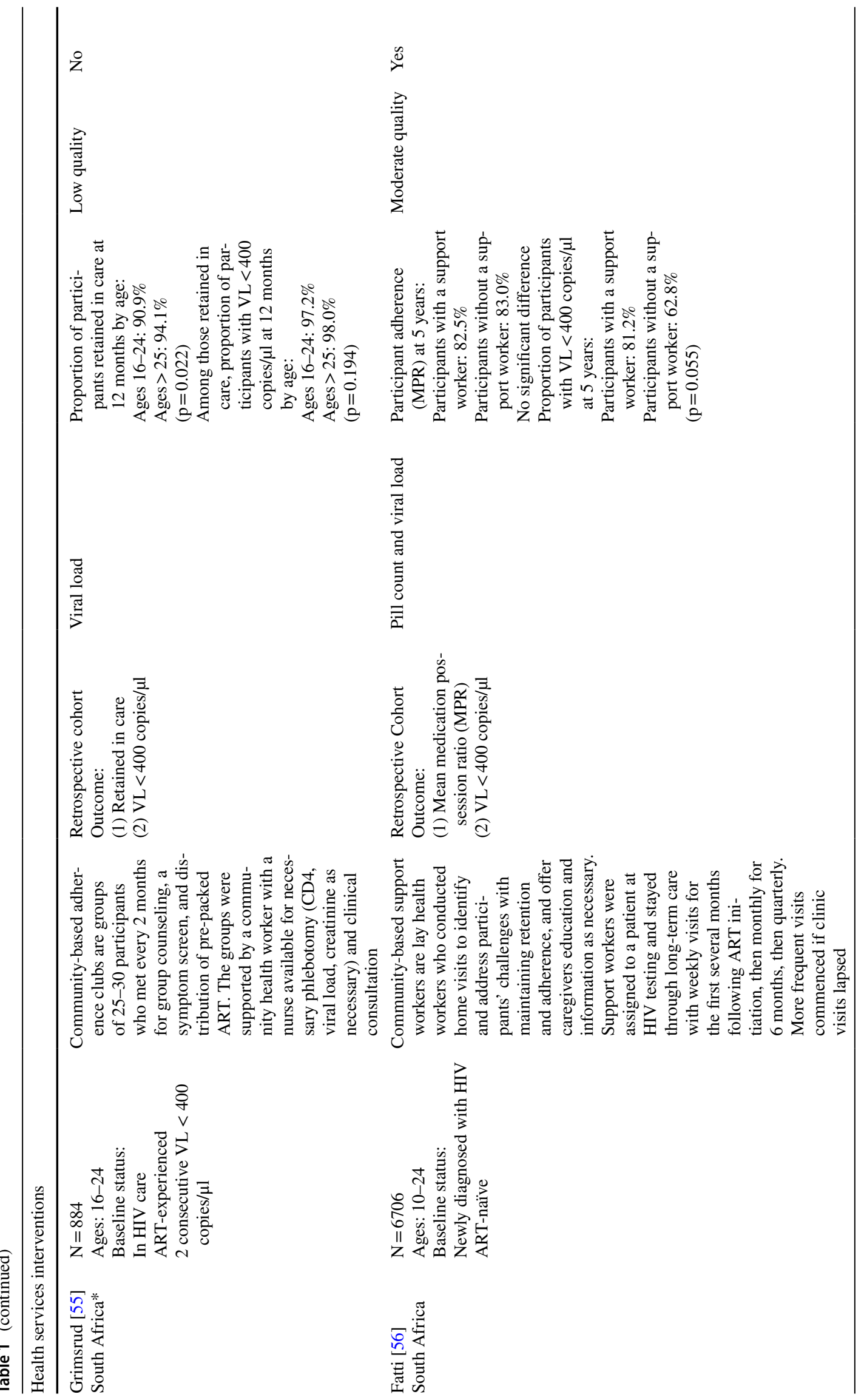




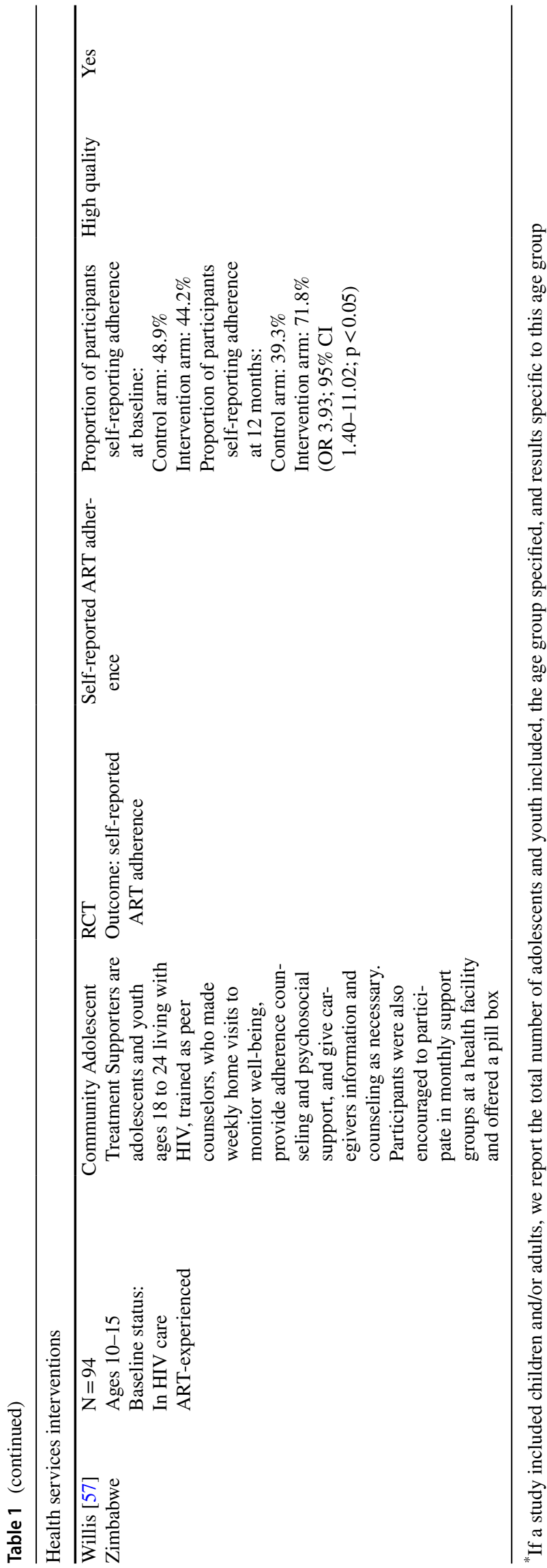

health worker [56] or peer counselor [57]. In the first, a community-based support worker (i.e. lay health worker) made weekly home visits to provide additional individualized adherence support. As participants became stable, frequency of home visits decreased to monthly and then quarterly, but increased again in the event of any clinic visit lapses [56). In the second, peer counselors (i.e. trained adolescents and youth ages 18 to 24 years living with HIV) referred to as 'community adolescent treatment supporters' provided adherence counseling and psychosocial support via weekly home visits [57].

\section{Patient-Specific Interventions}

\section{Study Design, Outcomes, and Participant Eligibility}

Of the three studies which evaluated patient-level interventions, two used experimental designs and one entailed a retrospective cohort study. The study evaluating text messaging used a 3-arm RCT to compare text messaging and text messaging with the option to respond to standard care (no text messaging). The primary outcome was mean ART adherence over the 48-week study period which was measured using medication event monitoring systems (MEMS) and defined as the ratio of recorded bottle openings to the number of prescribed bottle openings [51]. Eligible patients were 15 to 22 years of age, in care at an HIV care facility, and prescribed ART. The study evaluating a participant savings account used a 2-arm RCT comparing the intervention to standard care. The primary outcome was $\mathrm{VL}<40$ copies/ $\mu \mathrm{l} 24$ months after study enrollment [52]. Eligible patients were ages 10 to 16 years of age, in care at an HIV care facility, and prescribed ART. The study evaluating monthly intensive adherence counseling sessions used a retrospective cohort design and no comparison group. The primary outcome was $\mathrm{VL}<1000$ copies/ $\mu \mathrm{l}$ measured within 180 days of completing the 3 sessions [53]. Eligible patients were ages 10 to 19 years of age, in care at an HIV care facility, prescribed ART and had a VL>1000 copies/ $\mu$ l.

\section{Intervention Effectiveness}

None of the interventions in the three studies significantly improved ART adherence or VL outcomes in their primary analyses (all GRADE level 4). SMS text messaging did not significantly improve ART adherence over a 48-week period. Mean adherence (percentage of prescribed doses taken) was $64 \%$ in the group that received text messages $(\mathrm{p}=0.27$ compared to control), $61 \%$ in the group that received text messages with the option to respond $(\mathrm{p}=0.15$ compared to control), and $67 \%$ in the control group [51]. The study comparing participant savings accounts to standard care also showed no statistically significant difference in viral 
Fig. 2 Types of health services interventions

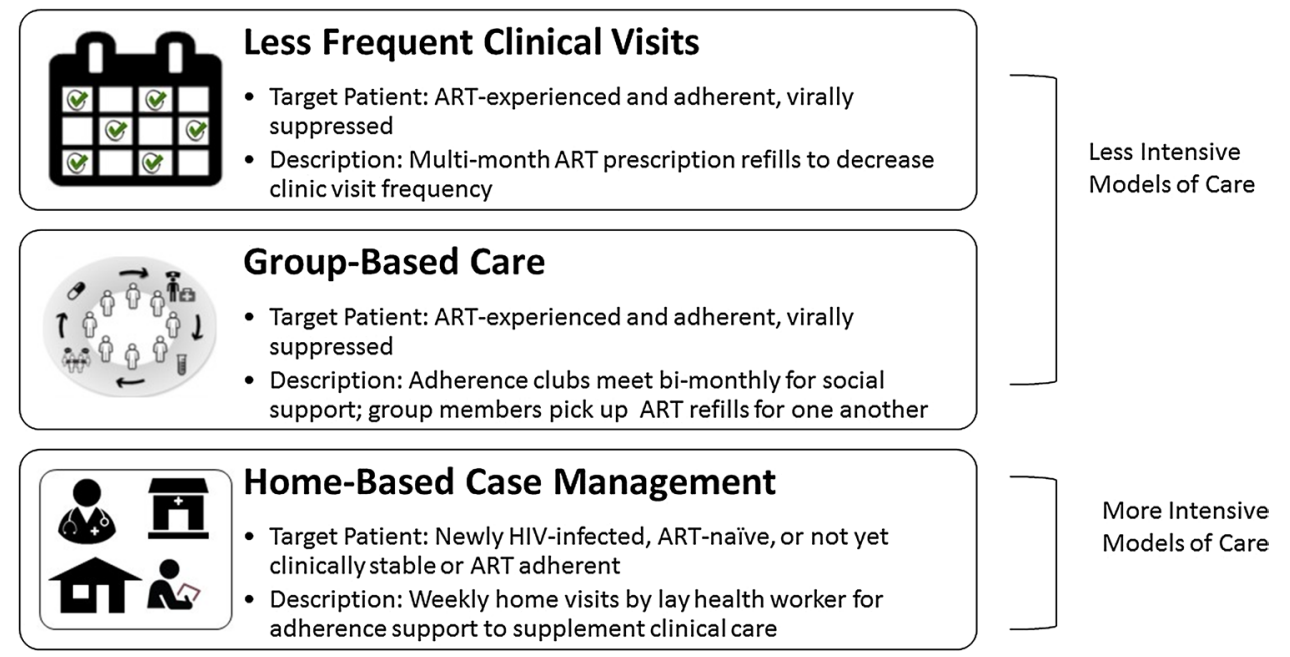

suppression between arms. The proportion of participants with a VL $<40$ copies/ $\mu$ at 24 months did not significantly differ by arm $-65.9 \%$ in the intervention arm and $63.4 \%$ in the control arm [52]. The observational study of intensive adherence counseling also observed low rates of VL suppression post-counseling. Among 192 adolescents included in the study, $117(60 \%)$ had a repeat VL measurement after 3 counseling sessions and 34/117 (29\%) achieved a VL $<1000$ copies/ $\mu \mathrm{l}[53]$.

\section{Health Services Interventions}

\section{Study Design, Outcomes, and Participant Eligibility}

The four studies evaluating health services interventions included three retrospective cohort studies and one RCT. The study evaluating multi-month ART prescriptions was a retrospective cohort study and used programmatic data to report outcomes of children and adolescents who transitioned to this less intensive model of care after they were deemed clinically stable and ART adherent, defined as having an improving CD4 + cell count or CD4\% or a VL $<400$ copies/ $\mu \mathrm{l}$ and pharmacy pill count $>95 \%$. The primary outcome $-\mathrm{VL}<400$ copies/ $\mu \mathrm{l}$ - was compared between age groups ( $<1$ year, 1-4 years, 5-9 years, 10-14 years, and 15-19 years) [54]. The group-based intervention study evaluating community-based adherence clubs was a retrospective cohort study among patients who self-reported ART adherence, had been on ART for $>12$ months, and had 2 consecutive VL measurements $<400$ copies/ $\mu$ l. Outcomes were maintaining a $\mathrm{VL}<400$ copies/ $\mu$ land viral rebound at 12 months from enrollment and were compared between age groups (16-24 years and $\geq 25$ years) [55]. Since eligibility for both of these studies required established ART adherence and clinical stability, these evaluations aimed to determine if adolescents and youth could maintain a stable status after shifting to a less intensive model of care.

The two studies of health service interventions which evaluated a more intensive model of care-home-based case management in addition to regular clinical care - included one retrospective cohort study and one RCT. The study evaluating the community-based support worker intervention examined a retrospective cohort of adolescents and youth ages 10 to 24 years who were newly diagnosed with HIV and were ART-naïve. Outcomes included mean medication possession ratio (ratio of days of dispensed medication to days between pharmacy visits) and VL $<400$ copies/ $\mu 1$ assessed at 3 and 5 years from initiating ART. Outcomes were compared to participants who did not receive support over the same 5 year study period [56]. The study evaluating community adolescent treatment supporters was a two-arm RCT among adolescents ages 10 to 15 years who were in care at an HIV care facility and prescribed ART. Participants were randomized to the treatment supporter intervention or standard care and the primary outcome - self-reported ART adherence_was compared by study arm [57].

\section{Intervention Effectiveness}

Both studies of less intensive models of care-multi-month ART prescriptions [54] and group-based care [55]—-showed a relatively high proportion of adolescents and youth maintained a VL $<400$ copies/ $\mu$ l over study follow-up (75\% and $97.2 \%$, respectively) (GRADE level 2). However in both studies, compared to children and adults, adolescents and youth were at higher risk for viral rebound (i.e. VL $>400$ copies/ $\mu \mathrm{l})$. In the study evaluating multi-month prescriptions, at baseline approximately $85 \%$ of children (ages 1-9 years) and $80 \%$ of adolescents (ages 10-19 years) had a VL $<400$ copies/ $\mu$ l. Over 60 months of follow-up with annual VL measurements, this proportion remained steady 
among children, but decreased among adolescents to approximately 75\% [54]. In the study of community-based adherence clubs, the proportion of adolescents (ages 16-24 years) retained in care at 12 months was significantly lower than that observed among adults (ages $\geq 25$ years) $-90.9 \%$ vs. $94.1 \%$, respectively $(\mathrm{p}=0.022)$. Among those retained in care, the proportion of participants with VL $<400$ copies/ $\mu \mathrm{l}$ was similar $-97.2 \%$ of adolescents and $98.0 \%$ of adults $(\mathrm{p}=0.194)$ —but in adjusted analyses, adolescents were at significantly higher risk of experiencing viral rebound compared to adults (adjusted hazard ratio: $2.24 ; 95 \%$ CI $1.0-5.04)$ [55].

The two studies examining intensified models of carehome-based case management $[56,57]$ — were the sole studies included in our review which improved ART adherence and viral suppression. The community-based support worker intervention which involved home visits by a lay health support worker, found that 5 years from initiating ART, 81.2\% of participants receiving the support intervention achieved a $\mathrm{VL}<400$ copies/ $\mu$ l compared to $62.8 \%$ among those not receiving support (adjusted odds ratio: $0.24 ; 95 \% \mathrm{CI}$ : 0.06-1.03; $\mathrm{p}=0.055$ ) (GRADE level 4). In the RCT examining the community adolescent treatment supporter intervention which involved home visits from a peer counselor also living with HIV, 12-months from study enrollment, $71.8 \%$ of participants in the intervention arm self-reported ART adherence compared to $39.3 \%$ receiving standard care $(\mathrm{p}<0.05)($ GRADE level 3).

\section{Additional Studies in High Income Countries}

There were four additional studies which met inclusion criteria but were conducted in high income settings. Two studies evaluated intensive individual or group counseling in the United States. The Positive Strategies to Enhance ProblemSolving Skills (STEPS) intervention consisted of five onehour counseling sessions rooted in cognitive-behavioral theory and motivational interviewing skills administered by a master's or doctoral-level clinician [58]. In a pilot RCT including participants self-reporting $<90 \%$ adherence at baseline, 14 participants were randomized to the STEPS intervention or standard care. After 4 months, mean ART adherence among participants randomized to STEPS increased by $13 \%$, and decreased by $26 \%$ among participants randomized to standard care, measured by MEMS data. No statistical comparison was conducted for this pilot RCT. The ACCEPT intervention was a group-based educational intervention including topics on stigma, disclosure, healthy relationships, and life planning [59]. In an RCT including 103 adolescents and youth newly diagnosed with HIV, participants in the ACCEPT intervention arm had a 2.33 greater likelihood of self-reported ART adherence at 12 months than those in the control arm $(\mathrm{p}=0.005)$.
Two studies evaluating technology-driven interventions showed statistically significant improvements in VL suppression. In a prospective cohort study conducted in Argentina, an intervention of twice-monthly private messaging through social media was evaluated among adolescents and youth with 2 consecutive VL measurements $>1000$ copies/ $\mu$ l. Twenty-two participants were enrolled and at 32 weeks, $64 \%$ achieved a $\mathrm{VL}<1000$ copies/ $\mu \mathrm{l}$ [60]. An RCT conducted in the United States included 66 participants ages 14-26 years with a detectable VL at baseline and evaluated an iPhone game designed around themes of 'fighting' or 'destroying' HIV in the body by taking ART. After 16-weeks, among participants who had newly initiated ART, the decrease in mean $\log$ VL of participants in the intervention arm (3.63-0.93) was significantly greater than the decrease in mean log VL of participants in the control arm (3.94-1.53) $(\mathrm{p}=0.04)$ [61].

\section{Discussion}

This systematic review identified seven studies published between 2015 and 2019 which evaluated a patient-level or health service intervention to improve ART adherence among adolescents and youth living with HIV ages 10-24 years in a LMIC. This expands upon a prior review of the same topic examining studies published between 2003 and 2015 [42], none of which were conducted in a LMIC. Among the seven studies in our review, three employed experimental designs appropriately powered to detect intervention effects, and five included a VL outcome, an objective biomarker of adherence [31]. The increase in the number and location of studies observed compared to the earlier review indicates a positive shift of focus to identify interventions to improve outcomes in regions with the greatest HIV burden. Additional high-quality evidence should be emerging soon from four ongoing RCTs we identified, two from sub-Saharan Africa [62-65].

The outcomes of the interventions evaluated indicate that ART adherence and viral suppression among adolescents and youth remain a major challenge. None of the three studies evaluating patient-level interventions, including two RCTs contributing high-quality evidence, showed improvement in either outcome. Adolescents and youth face a variety of barriers to ART adherence, which evolve as they develop physically, emotionally, and socially. Interventions designed to target a single, specific challenge, such as forgetfulness, may be insufficient. For example, the text message intervention was very narrow in scope-no additional counseling was done via text message and the messages were only sent weekly, so did not serve as a daily reminder for taking ART medication. Further research on combination interventions such as incorporating text messaging within a larger package of services is warranted, 
particularly as such combination intervention strategies have been effective among adults [66, 67].

The majority of studies reviewed focused on how HIV services were delivered to adolescents or youth [54-57]. The two studies on less intensive models of care indicated that adolescents and youth may be less likely to remain clinically stable as they were more likely to experience viral rebound than children or adults in both studies. Additional studies show that among adolescents and youth who do achieve VL suppression, only 50\% maintain this for one year [68]. Given adolescence itself is a dynamic period marked by constant development and changes to risk factors, and adolescents and youth are known to have suboptimal viral suppression outcomes, the concept of 'stable' adolescents and youth may be misleading.

Conversely, the more intensive HIV care models were the most effective interventions. Home-based case management interventions, which provided additional counseling to identify and address specific barriers which may not be recognized in the clinical setting, showed improved adherence and viral suppression outcomes. Individualized care and treatment within a larger health system may be the most efficient way to identify multiple or evolving challenges and then respond with a custom combination strategy. For example, if an adolescent or youth faces family discrimination, inability to miss school for clinic appointments, and lack of a trusted clinician relationship, the case-management intervention can target this specific barrier combination - family and social isolation, structural challenges of clinic-based care, and poor provider relationship [69, 70].

Several methodological limitations in the studies included should be noted. While community adolescent treatment supporters significantly improved outcomes [57], ART adherence was ascertained through self-report which may be subject to social desirability bias artificially leading to a positive effect. More objective adherence measures such as MEMS, drug levels or VL outcomes could avoid bias. The community-based support worker intervention also showed improved outcomes [56], but assignment to the intervention was not randomized. Patients received the intervention based on availability of support workers in the area, thus unmeasured confounding cannot be ruled out. For example, support workers may have only been available in more developed or easier-to-access locations. Further, as this intervention was evaluated using existing clinical data in a retrospective cohort design, data on the quality and consistency of intervention implementation is lacking, including frequency of home visits, type of support provided, and content of counseling. Lastly, a majority of studies report results just 1-year from implementing the intervention [50-52, 54, 56]. Evaluation of an intervention's effect over a longer period, particularly for new models of HIV care, could provide information on the long-term durability of an intervention on participant outcomes.

An additional limitation is the inability to identify adolescents and youth who have adequate ART adherence but are not virally suppressed, likely because of the presence of ART drug resistance. For participants who are ART-naïve or who have changed ART regimens, VL outcomes can accurately reflect improved ART adherence, but those who may have acquired ART drug resistance are unlikely to be VL suppressed even with improved ART adherence [71]. Four of the seven studies in this review only report VL outcomes and not an ART adherence measurement and only one is limited to ART-naïve participants [56]. Reporting the ART regimen prescribed could begin to address this limitation since research shows certain regimens are more likely to produce resistance than others $[72,73]$. Only one study in this review included data on participants' prescribed ART regimen [53].

Some interventions implemented in a high income setting improved outcomes among adolescents and youth [58-61] and may be feasibly adapted for use in resource-limited settings. Those utilizing social media or smart phone applications show promise given rapidly increasing access to internet and mobile phones among young people in LMICs [74]. Conversely, implementing interventions involving cognitive behavioral theory and motivational interviewing in highburden resource-limited settings is unlikely due to the high level of counselor training (i.e. implemented by $\mathrm{PhD}$-level practitioner) and amount of time required for individualized counseling.

Medication-related barriers-side-effects and ease of daily dosing - are critical aspects of adherence and require simplification. We did not include studies which address such barriers through use of alternative regimens or formulations. Use of more potent and/or better tolerated ART regimens such as integrase inhibitors, long-acting and injectable regimens [75-77] have shown promise among adults and should be examined among adolescents and youth. Other medication-related interventions among adolescents and youth such as weekends off regimens have been shown to be non-inferior [78], and single-tablet regimens have achieved significantly higher VL suppression rates than multi-tablet regimens [79]. Medication-related interventions implemented in parallel with health services interventions could significantly and sustainably impact adolescent and youth ART adherence.

\section{Conclusion}

ART adherence and viral suppression outcomes remain a major challenge among adolescents and youth living with HIV in LMICs. Recent studies of interventions to improve ART adherence among this population show inconsistent 
effects, highlighting the need for additional, innovative approaches which specifically target the needs of adolescents and youth. To date, individually-targeted interventions have not shown significant effects on adherence, but health services interventions which enhance clinic-based care with home-based care, appear promising. There is a clear need for appropriately powered studies examining combination interventions. Standardizing the key outcomes applied across these studies can streamline limited resources, maximize the impact of research, and yield effective interventions to improve health outcomes for adolescents and youth living with HIV.

Open Access This article is licensed under a Creative Commons Attribution 4.0 International License, which permits use, sharing, adaptation, distribution and reproduction in any medium or format, as long as you give appropriate credit to the original author(s) and the source, provide a link to the Creative Commons licence, and indicate if changes were made. The images or other third party material in this article are included in the article's Creative Commons licence, unless indicated otherwise in a credit line to the material. If material is not included in the article's Creative Commons licence and your intended use is not permitted by statutory regulation or exceeds the permitted use, you will need to obtain permission directly from the copyright holder. To view a copy of this licence, visit http://creativecommons.org/licenses/by/4.0/.

\section{References}

1. Adejumo OA, Malee KM, Ryscavage P, Hunter SJ, Taiwo BO. Contemporary issues on the epidemiology and antiretroviral adherence of HIV-infected adolescents in sub-Saharan Africa: a narrative review. J Int AIDS Soc. 2015;18:20049.

2. Salou M, Dagnra AY, Butel C, et al. High rates of virological failure and drug resistance in perinatally HIV-1-infected children and adolescents receiving lifelong antiretroviral therapy in routine clinics in Togo. J Int AIDS Soc. 2016;19(1):20683.

3. Belzer ME, Fuchs DN, Luftman GS, Tucker DJ. Antiretroviral adherence issues among HIV-positive adolescents and young adults. J Adolesc Health. 1999;25(5):316-9.

4. Lamb MR, Fayorsey R, Nuwagaba-Biribonwoha H, et al. High attrition before and after ART initiation among youth (15-24 years of age) enrolled in HIV care. AIDS. 2014;28(4):559-68.

5. Nachega JB, Mills EJ, Schechter M. Antiretroviral therapy adherence and retention in care in middle-income and low-income countries: current status of knowledge and research priorities. Current Opinion in HIV and AIDS. 2010;5(1):70-7.

6. Paterson DL, Swindells S, Mohr J, et al. Adherence to protease inhibitor therapy and outcomes in patients with HIV infection. Ann Intern Med. 2000;133(1):21-30.

7. UNICEF. Children and AIDS. Statistical Update. New York: UNICEF. 2015. Available from https://data.unicef.org/wp-conte nt/uploads/2015/12/2015-Children-Adolescents-and-AIDS-Stati stical-Update-Executive-Summary_244.pdf. Accessed 15 Aug 2019.

8. UNICEF. Adolescent HIV Prevention. UNICEF Data. 2018. Available from https://data.unicef.org/topic/hivaids/adolescent s-young-people/. Accessed 15 Aug 2019.
9. Haynes RB, Ackloo E, Sahota N, McDonald HP, Yao X. Interventions for enhancing medication adherence. Cochrane Database Syst Rev. 2008;2:Cd000011.

10. Haynes RB, Sackett DL, Taylor DW. How to detect and manage low patient compliance in chronic illness. Geriatrics. 1980;35(1):91.

11. Morrissey EC, Durand H, Nieuwlaat R, et al. Effectiveness and content analysis of interventions to enhance medication adherence in hypertension: a systematic review and meta-analysis protocol. Syst Rev. 2016;5:96.

12. Nieuwlaat R, Wilczynski N, Navarro T, et al. Interventions for enhancing medication adherence. Cochrane Database Syst Rev. 2014;11:Cd000011.

13. Fogarty L, Roter D, Larson S, Burke J, Gillespie J, Levy R. Patient adherence to HIV medication regimens: a review of published and abstract reports. Patient Educ Couns. 2002;46(2):93-108.

14. Ammassari A, Antinori A, Aloisi MS, et al. Depressive symptoms, neurocognitive impairment, and adherence to highly active antiretroviral therapy among HIV-infected persons. Psychosomatics. 2004;45(5):394-402.

15. Ammassari A, Trotta MP, Murri R, et al. Correlates and predictors of adherence to highly active antiretroviral therapy: overview of published literature. J Acquir Immune Defic Syndr. 2002;31(Suppl 3):S123-S127127.

16. Ammassari AA, Starace F, Aloisi MS, et al. Medication adherence among HIV+ adults: effects of cognitive dysfunction and regimen complexity. Neurology. 2003;61(5):723-4.

17. Abubakar A, Van de Vijver FJ, Fischer R, et al. 'Everyone has a secret they keep close to their hearts': challenges faced by adolescents living with HIV infection at the Kenyan coast. BMC Public Health. 2016;16:197.

18. Choudhury S, Blakemore SJ, Charman T. Social cognitive development during adolescence. Soc Cognit Affect Neurosci. 2006;1(3):165-74.

19. Willoughby T, Good M, Adachi PJC, Hamza C, Tavernier R. Examining the link between adolescent brain development and risk taking from a social-developmental perspective. Brain Cogn. 2013;83:315-23.

20. Yakubu I, Salisu WJ. Determinants of adolescent pregnancy in sub-Saharan Africa: a systematic review. Reprod Health. 2018;15(1):15.

21. Tanney MR, Naar-King S, MacDonnel K. Depression and stigma in high-risk youth living with HIV: a multi-site study. J Pediatr Health Care. 2012;26(4):300-5.

22. Mutumba M, Musiime V, Lepkwoski JM, et al. Examining the relationship between psychological distress and adherence to anti-retroviral therapy among Ugandan adolescents living with HIV. AIDS Care. 2016;28(7):807-15.

23. Petersen I, Bhana A, Myeza N, et al. Psychosocial challenges and protective influences for socio-emotional coping of HIV+ adolescents in South Africa: a qualitative investigation. AIDS Care. 2010;22(8):970-8.

24. Laughton B, Cornell M, Boivin M, Van Rie A. Neurodevelopment in perinatally HIV-infected children: a concern for adolescence. J Int AIDS Soc. 2013;16:18603.

25. Smith R, Chernoff M, Williams PL, et al. Impact of HIV severity on cognitive and adaptive functioning during childhood and adolescence. Pediatr Infect Dis J. 2012;31(6):592-8.

26. Kalichman SC, Kalichman MO, Cherry C. Forget about forgetting: structural barriers and severe non-adherence to antiretroviral therapy. AIDS Care. 2016;29:1-5.

27. Krummenacher I, Spencer B, Du Pasquier S, Bugnon O, Cavassini M, Schneider MP. Qualitative analysis of barriers and facilitators encountered by HIV patients in an ART adherence programme. Int J Clin Pharm. 2014;36(4):716-24. 
28. Shubber Z, Mills EJ, Nachega JB, et al. Patient-reported barriers to adherence to antiretroviral therapy: a systematic review and meta-analysis. PLoS Med. 2016;13(11):e1002183.

29. Ankrah DN, Koster ES, Mantel-Teeuwisse AK, Arhinful DK, Agyepong IA, Lartey M. Facilitators and barriers to antiretroviral therapy adherence among adolescents in Ghana. Patient Prefer Adher. 2016;10:329-37.

30. Soboka M, Feyissa GT. The effectiveness of counseling, material support and/or nutritional supplementation on improving adherence to anti-retroviral therapy and clinical outcomes among HIV patients: a systematic review of quantitative evidence protocol. JBI Database Syst Rev Implement Rep. 2015;13(7):142-52.

31. Ferrand RA, Briggs D, Ferguson J, et al. Viral suppression in adolescents on antiretroviral treatment: review of the literature and critical appraisal of methodological challenges. Trop Med Int Health. 2016;21(3):325-33.

32. Mutwa PR, Van Nuil JI, Asiimwe-Kateera B, et al. Living situation affects adherence to combination antiretroviral therapy in HIV-infected adolescents in Rwanda: a qualitative study. PLoS ONE. 2013;8(4):e60073.

33. Musumari PM, Feldman MD, Techasrivichien T, Wouters E, OnoKihara M, Kihara M. "If I have nothing to eat, I get angry and push the pills bottle away from me": a qualitative study of patient determinants of adherence to antiretroviral therapy in the Democratic Republic of Congo. AIDS Care. 2013;25(10):1271-7.

34. Rudy BJ, Murphy DA, Harris DR, Muenz L, Ellen J. Patientrelated risks for nonadherence to antiretroviral therapy among HIV-infected youth in the United States: a study of prevalence and interactions. AIDS Patient Care and STDs. 2009;23(3):185-94.

35. Hermetet-Lindsay KD, Correia KF, Williams PL, et al. Contributions of disease severity, psychosocial factors, and cognition to behavioral functioning in US youth perinatally exposed to HIV. AIDS Behav. 2016;21:2703-15.

36. Daigle GT, Jolly PE, Chamot EA, et al. System-level factors as predictors of adherence to clinical appointment schedules in antiretroviral therapy in Cambodia. AIDS Care. 2015;27(7):836-43.

37. Hudelson C, Cluver L. Factors associated with adherence to antiretroviral therapy among adolescents living with HIV/AIDS in low- and middle-income countries: a systematic review. AIDS Care. 2015;27(7):805-16.

38. Murphy DA, Sarr M, Durako SJ, Moscicki AB, Wilson CM, Muenz LR. Barriers to HAART adherence among human immunodeficiency virus-infected adolescents. Arch Pediatr Adolesc Med. 2003;157(3):249-55.

39. Murphy DA, Wilson CM, Durako SJ, Muenz LR, Belzer M. Antiretroviral medication adherence among the REACH HIV-infected adolescent cohort in the USA. AIDS Care. 2001;13(1):27-40.

40. Buchanan AL, Montepiedra G, Sirois PA, et al. Barriers to medication adherence in HIV-infected children and youth based on selfand caregiver report. Pediatrics. 2012;129(5):e1244-e12511251.

41. Holtzman CW, Shea JA, Glanz K, et al. Mapping patient-identified barriers and facilitators to retention in HIV care and antiretroviral therapy adherence to Andersen's Behavioral Model. AIDS Care. 2015;27(7):817-28

42. Shaw S, Amico KR. Antiretroviral therapy adherence enhancing interventions for adolescents and young adults 13-24 years of age: a review of the evidence base. J Acquir Immune Defic Syndr. 2016;72(4):387-99.

43. Dowshen N, Kuhns LM, Johnson A, Holoyda BJ, Garofalo R. Improving adherence to antiretroviral therapy for youth living with HIV/AIDS: a pilot study using personalized, interactive, daily text message reminders. J Med Internet Res. 2012;14(2):e51.

44. Belzer ME, Naar-King S, Olson J, et al. The use of cell phone support for non-adherent HIV-infected youth and young adults: an initial randomized and controlled intervention trial. AIDS Behav. 2014;18(4):686-96.

45. Naar-King S, Outlaw AY, Sarr M, et al. Motivational Enhancement System for Adherence (MESA): pilot randomized trial of a brief computer-delivered prevention intervention for youth initiating antiretroviral treatment. J Pediatr Psychol. 2013;38(6):638-48.

46. The World by Income and Region. The World Bank. 2017. Available from: https://datatopics.worldbank.org/world-developmen t-indicators/the-world-by-income-and-region.html. Accessed 15 Aug 2019.

47. Shamseer L, Moher D, Clarke M, Ghersi D, Liberati A, Petticrew M, Shekelle P, Stewart L, PRISMA-P Group. Preferred reporting items for systematic review and meta-analysis protocols (PRISMA-P) 2015: elaboration and explanation. BMJ. 2015 2;349:g7647.

48. Antiretroviral Therapy for HIV Infection in Infants and Children: Towards Universal Access: Recommendations for a Public Health Approach. Geneva: World Health Organization; 2010. Available from: https://www.ncbi.nlm.nih.gov/books/NBK13 8585/. Accessed 15 Aug 2019.

49. The Global Fund. A Toolkit for Health Facilities: Differentiated Care for HIV and Tuberculosis. Geneva, Switzerland; 2015. Available from: https://www.theglobalfund.org/media/2569/ core_differentiatedcare_toolkit_en.pdf. Accessed 15 Aug 2019.

50. Grimsrud A, Bygrave H, Doherty M, et al. Reimagining HIV service delivery: the role of differentiated care from prevention to suppression. J Int AIDS Soc. 2016;19(1):21484.

51. Linnemayr S, Huang H, Luoto J, et al. Text Messaging for Improving Antiretroviral Therapy Adherence: No Effects After 1 Year in a Randomized Controlled Trial Among Adolescents and Young Adults. Am J Public Health. 2017;107(12):1944-50.

52. Bermudez LG, Ssewamala FM, Neilands TB, et al. Does Economic Strengthening improve viral suppression among adolescents living with HIV? results from a cluster randomized trial in Uganda. AIDS Behav. 2018;22(11):3763-72.

53. Nasuuna E, Kigozi J, Babirye L, Muganzi A, Sewankambo NK, Nakanjako D. Low HIV viral suppression rates following the intensive adherence counseling (IAC) program for children and adolescents with viral failure in public health facilities in Uganda. BMC Public Health. 2018;18(1):1048.

54. Kim M, Wanless RS, Caviness C, et al. Multi-month prescription of antiretroviral therapy amongst children and adolescents: experiences from the Baylor International Pediatric AIDS initiative (BIPAI) in six African countries. J Acquir Immune Defic Syndr. 2018;78(Suppl 2):S71-80.

55. Grimsrud A, Lesosky M, Kalombo C, Bekker LG, Myer L. Implementation and operational research: community-based adherence clubs for the management of stable antiretroviral therapy patients in Cape Town, South Africa: a cohort study. J Acquir Immune Defic Syndr. 2016;71(1):e16-23.

56. Fatti G, Jackson D, Goga AE, et al. The effectiveness and costeffectiveness of community-based support for adolescents receiving antiretroviral treatment: an operational research study in South Africa. J Int AIDS Soc. 2018;21(Suppl):1.

57. Willis N, Milanzi A, Mawodzeke M, et al. Effectiveness of community adolescent treatment supporters (CATS) interventions in improving linkage and retention in care, adherence to ART and psychosocial well-being: a randomised trial among adolescents living with HIV in rural Zimbabwe. BMC Public Health. 2019;19(1):117.

58. Mimiaga MJ, Bogart LM, Thurston IB, et al. positive strategies to enhance problem-solving skills (STEPS): a pilot randomized, controlled trial of a multicomponent, technology-enhanced, customizable antiretroviral adherence intervention for HIV-infected adolescents and young adults. AIDS Patient Care and STDs. 2019;33(1):21-4. 
59. Hosek SG, Harper GW, Lemos D, et al. Project ACCEPT: Evaluation of a group-based intervention to improve engagement in care for youth newly diagnosed with HIV. AIDS Behav. 2018;22(8):2650-61.

60. Stankievich E, Malanca A, Foradori I, Ivalo S, Losso M. Utility of mobile communication devices as a tool to improve adherence to antiretroviral treatment in HIV-infected children and young adults in Argentina. Pediatr Infect Dis J. 2018;37(4):345-8.

61. Whiteley L, Brown LK, Mena L, Craker L, Arnold T. Enhancing health among youth living with HIV using an iPhone game. AIDS Care. 2019;30:1-13.

62. Belzer ME, MacDonell KK, Ghosh S, et al. Adaptive antiretroviral therapy adherence interventions for youth living with HIV through text message and cell phone support with and without incentives: protocol for a sequential multiple assignment randomized trial (SMART). JMIR Res Protoc. 2018;7(12):e11183.

63. Mavhu W, Willis N, Mufuka J, et al. Evaluating a multi-component, community-based program to improve adherence and retention in care among adolescents living with HIV in Zimbabwe: study protocol for a cluster randomized controlled trial. Trials. 2017;18(1):478.

64. Mimiaga MJ, Kuhns LM, Biello KB, et al. Positive STEPS - a randomized controlled efficacy trial of an adaptive intervention for strengthening adherence to antiretroviral HIV treatment among youth: study protocol. BMC Public Health. 2018;18(1):867.

65. Ekwunife OI, Anetoh MU, Kalu SO, Ele PU, Eleje GU. Conditional economic incentives and motivational interviewing to improve adolescents' retention in HIV care and adherence to antiretroviral therapy in Southeast Nigeria: study protocol for a cluster randomised trial. Trials. 2018;19(1):710.

66. Elul B, Lamb MR, Lahuerta M, et al. A combination intervention strategy to improve linkage to and retention in HIV care following diagnosis in Mozambique: A cluster-randomized study. PLoS Med. 2017;14(11):e1002433.

67. McNairy ML, Lamb MR, Gachuhi AB, et al. Effectiveness of a combination strategy for linkage and retention in adult HIV care in Swaziland: The Link4Health cluster randomized trial. PLoS Med. 2017;14(11):e1002420.

68. Murphy DA, Belzer M, Durako SJ, Sarr M, Wilson CM, Muenz LR. Longitudinal antiretroviral adherence among adolescents infected with human immunodeficiency virus. Arch Pediatr Adolesc Med. 2005;159(8):764-70.

69. Frieson Bonaparte K, Graves C, Farber, E, Gillespie S, Hussen S, Thomas-Seaton L, Chakraborty R, Camacho-Gonzalez A. Metropolitan Atlanta community adolescent rapid testing initiative: the impact of motivational interviewing and intensive case management on the psychosocial and clinical care outcomes of adolescents and young adults with HIV. Psychotherapy (Chic). 2019; pii: 2019-62569-001.

70. Broaddus MR, Hanna CR, Schumann C, Meier A. "She makes me feel that I'm not alone": linkage to Care Specialists provide social support to people living with HIV. AIDS Care. 2015;27(9):1104-7.

71. Nachega JB, Marconi VC, van Zyl GU, et al. HIV treatment adherence, drug resistance, virologic failure: evolving concepts. Infect Disord Drug Targets. 2011;11(2):167-74.

72. Giacomet V, Cossu MV, Capetti AF, Zuccotti G, Rizzardini G. An evaluation of elvitegravir plus cobicistat plus tenofovir alafenamide plus emtricitabine as a single-tablet regimen for the treatment of HIV in children and adolescents. Expert Opin Pharmacother. 2019;20(3):269-76.

73. Walmsley SL, Antela A, Clumeck N, et al. Dolutegravir plus abacavir-lamivudine for the treatment of HIV-1 infection. N Engl J Med. 2013;369(19):1807-18.

74. Ippoliti NB, L'Engle K. Meet us on the phone: mobile phone programs for adolescent sexual and reproductive health in lowto-middle income countries. Reprod Health. 2017;14(1):11.

75. Kerrigan D, Mantsios A, Gorgolas M, et al. Experiences with long acting injectable ART: a qualitative study among PLHIV participating in a Phase II study of cabotegravir + rilpivirine (LATTE-2) in the United States and Spain. PLoS ONE. 2018;13(1):e0190487.

76. Margolis DA, Gonzalez-Garcia J, Stellbrink HJ, et al. Longacting intramuscular cabotegravir and rilpivirine in adults with HIV-1 infection (LATTE-2): 96-week results of a randomised, open-label, phase $2 \mathrm{~b}$, non-inferiority trial. Lancet. 2017;390(10101):1499-510.

77. Markowitz M, Frank I, Grant RM, et al. Safety and tolerability of long-acting cabotegravir injections in HIV-uninfected men (ECLAIR): a multicentre, double-blind, randomised, placebocontrolled, phase 2a trial. Lancet HIV. 2017;4(8):e331-e34040.

78. Turkova A, Moore CL, Butler K, et al. Weekends-off efavirenzbased antiretroviral therapy in HIV-infected children, adolescents, and young adults (BREATHER): a randomised, open-label, noninferiority, phase 2/3 trial. Lancet HIV. 2016;3(9):e421-e430430.

79. Griffith DC, Farmer C, Gebo KA, et al. Uptake and virological outcomes of single- versus multi-tablet antiretroviral regimens among treatment-naive youth in the HIV Research Network. HIV Med. 2019;20(2):169-74.

Publisher's Note Springer Nature remains neutral with regard to jurisdictional claims in published maps and institutional affiliations.

\section{Affiliations}

\section{Lindsey K. Reif ${ }^{1,6}$. Elaine J. Abrams s,4,6 . Stephen Arpadi ${ }^{2,3,4} \cdot$ Batya Elul $^{6} \cdot$ Margaret L. McNairy ${ }^{1,5}$. Daniel W. Fitzgerald ${ }^{1} \cdot$ Louise Kuhn ${ }^{2,6}$}

Elaine J. Abrams

eja1@cumc.columbia.edu

Stephen Arpadi

sma2@columbia.edu

Batya Elul

be2124@cumc.columbia.edu

Margaret L. McNairy

mam9365@med.cornell.edu
Daniel W. Fitzgerald

dwf2001@med.cornell.edu

Louise Kuhn

1k24@cumc.columbia.edu

1 Center for Global Health, Department of Medicine, Weill Cornell Medicine, New York, NY, USA

2 Gertrude H. Sergievsky Center, Vagelos College of Physicians and Surgeons, Columbia University Irving Medical Center, New York, NY, USA 
3 ICAP At Columbia University, Mailman School of Public Health, Columbia University Irving Medical Center, New York, NY, USA

4 Department of Pediatrics, Vagelos College of Physicians and Surgeons, Columbia University Irving Medical Center, New York, NY, USA
5 Division of General Internal Medicine, Department of Medicine, Weill Cornell Medicine, New York, NY, USA

6 Department of Epidemiology, Mailman School of Public Health, Columbia University Irving Medical Center, New York, NY, USA 